\title{
L'apport de la sculpture à la compréhension des fonctions liturgiques
}

The contribution of sculpture to the understanding of liturgical functions

\section{Pascale Chevalier}

\section{(2)enEdition}

\section{Journals}

Édition électronique

URL : https://journals.openedition.org/cel/19564

DOl : $10.4000 /$ cel. 19564

ISSN : 2262-208X

\section{Éditeur}

École du Louvre

\section{Référence électronique}

Pascale Chevalier, «L'apport de la sculpture à la compréhension des fonctions liturgiques », Les Cahiers de l'École du Louvre [En ligne], 17 | 2021, mis en ligne le 18 novembre 2021, consulté le 13 décembre 2021. URL : http://journals.openedition.org/cel/19564 ; DOI : https://doi.org/10.4000/cel. 19564

Ce document a été généré automatiquement le 13 décembre 2021.

\section{cc) (†)}

Les Cahiers de l'École du Louvre sont mis à disposition selon les termes de la licence Creative Commons Attribution - Pas d'Utilisation Commerciale - Pas de Modification 4.0 International. 


\title{
L'apport de la sculpture à la compréhension des fonctions liturgiques
}

The contribution of sculpture to the understanding of liturgical functions

\author{
Pascale Chevalier
}

1 Au sein de l'espace ecclésial qui est né avec les premières basiliques chrétiennes au IV ${ }^{e}$ siècle, le mobilier liturgique était un des lieux de la sculpture. Autels, ciboria, cathèdres, chancels, ambons, fonts baptismaux... en pierre, en bois ou en stuc, étaient ainsi ornés, jusqu'à l'an Mil, de moulures et de sculptures au contenu plutôt aniconique et conforme au titre de ces journées, autrement dit à la fois «classique et barbare ». La majorité des combinaisons de décors reconnus appartiennent à des répertoires caractéristiques qui évoluèrent dans le temps tandis que se maintenaient des techniques peu variées. Ciselures, gravures, méplat, champlevé, bas-relief ou travail ajouré se développaient sur les limites matérielles de l'espace sacré, renforçaient le sens et embellissaient l'autel du sacrifice, le siège du célébrant, l'estrade d'où résonnait la parole... L'héritage antique détermina la morphologie spécifique et parlante de chaque meuble, l'implantation et la mise en page même des décors sculptés, dont l'évolution stylistique accompagna celle des autres médiums artistiques sur l'actuel territoire français. Outre les moulures, les ornements géométriques et végétaux, également hérités de l'Antiquité ou faisant partie des apports «barbares", ces sculptures portaient des messages visuels, symboliques ou clairement énoncés, adaptés à leur fonction et à l'espace ecclésial que les différents aménagements liturgiques jalonnaient, structuraient et explicitaient. 


\section{Les éléments sculptés liés aux fonctions liturgiques, le mobilier liturgique sculpté}

2 Les collections et exemples isolés de sculptures des IV $-\mathrm{X}^{\mathrm{e}}$ siècles conservés en France regorgent d'éléments plus ou moins complets appartenant à un meuble liturgique démantelé et souvent partiellement conservé. Ce que l'on nomme en effet mobilier liturgique ${ }^{1}$ correspond dans les faits, lorsque l'on considère les fragments sculptés, à des aménagements fixes, initialement ancrés dans les sols des édifices ou fixés sur des socles, des stylobates, des massifs maçonnés ${ }^{2}$.. Les parties moulurées et sculptées concernaient le meuble dans son ensemble, sa superstructure voire son seul revêtement. Ces meubles composites étaient en outre toujours "préfabriqués ", commandés et livrés en pièces détachées, destinées à être montées et fixées in situ ${ }^{3}$. Ils résultaient du montage de plusieurs pièces ornées ou non, associées selon des systèmes, des formes, des architectures définis à l'époque paléochrétienne et qui connurent dans les sept siècles considérés des évolutions diverses suivant les cas. Tous présentaient, quoi qu'il en soit de leur décor, une morphologie "parlante». Chaque meuble était immédiatement identifiable par son aspect général même, sa structure d'ensemble, qui annonçait en quelque sorte au clergé comme aux fidèles ce qu'il était et ce à quoi il servait. Dans le même ordre d'idées, les modénatures, les épannelages, les compositions architecturées, les formes mêmes et certains décors étaient «fonctionnels » ou plutôt résultaient du rôle principal d'un dispositif donné et du modèle fonctionnel antique qui l'avait inspiré (voir infra).

3 L'aménagement mobilier de l'abside - une banquette presbytérale maçonnée ou en bois - était très peu sculpté. Seule la cathèdre du principal ecclésiastique présent (évêque, abbé, simple prêtre...) portait quelquefois des ornements gravés ou en relief, sur les accoudoirs ou le dosseret. Cette sobriété sculpturale mettait en valeur le célébrant dans ce trône centré dans l'axe, mais surtout permettait d'exalter visuellement le meuble essentiel qu'était l'autel. Celui-ci imita à l'origine les tables de salle à manger romaines : un plateau mouluré porté par de minces supports dont les bases s'encastraient dans un socle ou dans le sol même du sanctuaire - la sculpture s'y concentrait sur la tranche de la mensa ou son rebord et sur les chapiteaux des colonnettes, comme dans l'Antiquité classique. On créa ensuite des autels-cippes ${ }^{4}$, récupérant (ou imitant et «christianisant » par le décor) des arae "païennes » ou des cippes funéraires ${ }^{5}$, qui constituèrent le support unique d'un plateau rectangulaire de dimensions réduites, mais toujours doté de la même bordure épaisse et moulurée ; ces stipites étaient ciselés parfois sur les quatre faces et la tranche de la mensa pouvait être sculptée. Vinrent enfin quelques autels-blocs et autels-caisses éventuellement encadrés aux angles de minces colonnettes ; outre les chapiteaux et les bases de ces dernières, le décor mouluré et sculpté de ce type d'autel était généralement plus détaillé sur la face tournée vers les fidèles, même si le revers et les plaques latérales portaient parfois une ornementation gravée ou sculptée. Indépendamment de leur typologie, un petit nombre d'autels était magnifié par un ciborium, dont la superstructure architecturée mettait en œuvre des épistyles ou des arcs portés par quatre colonnes; en plus des chapiteaux de supports de ce dais qui s'élevait à 3-4 m, les faces de ces arcs visibles depuis le sanctuaire et la nef étaient généralement sculptées. Composition architecturée, modénature et sculpture étaient, on le perçoit d'emblée, des aspects 
cruciaux de la caractérisation visuelle du meuble par excellence de l'eucharistie, autour duquel s'articulait la liturgie et le sanctuaire lui-même.

Fig. 1.

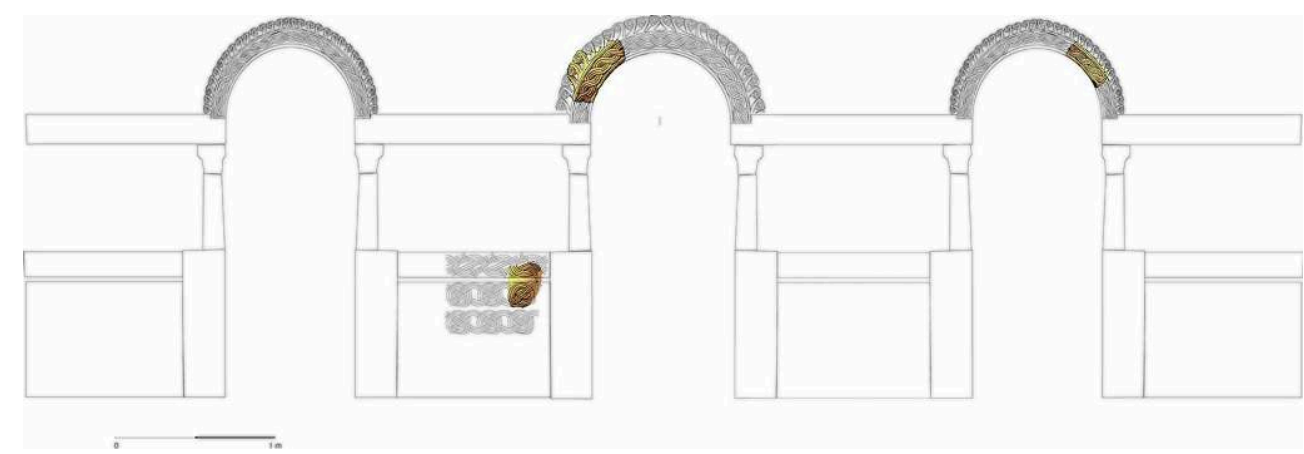

Restitution idéale de la pergula (chancel haut) en calcaire de Pouthumé près de Châtellerault ; les fragments sont issus d'une fouille Inrap dirigée par Thierry Cornec, del. A. Flammin.

(C) A. Flammin

4 Délimitant l'espace le plus sacré - celui du sanctuaire, réservé aux clercs -, le signalant au sein de l'édifice et le protégeant d'incursions intempestives, le chancel de pierre était un assemblage de plaques et de piliers sculptés haut d'1 $\mathrm{m}$, inspiré des barrières antiques. À compter au moins des VIII ${ }^{\mathrm{e}}$-IX $\mathrm{X}^{\mathrm{e}}$ siècles, les plaques furent encadrées par des piliers-colonnettes supportant à $2 \mathrm{~m}$ de hauteur environ des architraves et plus haut encore des arcs voire des tympans au-dessus des accès, formant ce que l'on nommait dans les espaces méditerranéens pergula (ainsi à Pouthumé6) (fig. 1). Le chancel formait pour les fidèles un écran que seul pouvait franchir leur regard, s'il ne s'arrêtait aux ornements sculptés qu'offrait la face tournée vers eux de la barrière de chœur. En effet, ce dispositif situé à l'interface entre espace des clercs et espace des laïcs offrait en général la plus grande surface disponible aux décors en relief ou gravés. En plus des parapets bordant une possible solea, sorte de couloir donnant accès au chœur depuis la nef ou reliant le chœur à un ambon, d'autres barrières et parapets existaient encore dans certaines églises ou dans leurs annexes liturgiques et funéraires (parapets de tribunes; enceinte autour d'une tombe sainte, ainsi dans l'exemple exceptionnel de la basilique paléochrétienne de la rue Malaval de Marseille ${ }^{7}$ ou dans le monument funéraire carolingien de Saint-Pons de Cimiez ${ }^{8}$, etc.) ; moulures et sculptures ornaient également ces autres barrières basses.

Dans la nef, le dernier meuble liturgique généralement sculpté était l'ambon, une plateforme ceinte d'un parapet qui pouvait s'étendre aussi à son accès ou ses accès. On trouvait encore quelques ciboria baptismaux, dont les supports reposaient autour de la piscine, parfois sur sa margelle polygonale ou cruciforme. Là encore le décor en relief concernait les chapiteaux et la superstructure d'architraves ou d'arcs. Enfin, un dais apparaissait quelquefois pour mettre en valeur une sépulture privilégiée avec le même type de répartition des reliefs.

6 Lorsque l'on ignore le contexte d'origine d'une des pièces d'un des différents assemblages mobiliers décrits plus haut, notamment son implantation précise dans l'édifice - et c'est le fait de la plupart des éléments sculptés du corpus SATHMA - et que la pièce examinée est très fragmentaire, il peut être compliqué de l'attribuer à l'un ou l'autre meuble liturgique. Cependant, son matériau, sa morphologie, tout particulièrement ses moulures et ses sculptures ainsi que les motifs mis en œuvre et 
leurs combinaisons, les dimensions même des éléments du décor en relief ou gravé, fournissent bien souvent des clefs de lecture et d'identification permettant de resituer le fragment considéré dans l'espace ecclésial.

\section{Mise en page de la sculpture entre héritages « antiques » et influx « barbares »}

7 Dans le cas des chapiteaux, les variations tiennent essentiellement au matériau employé, au choix du type de feuilles et de volutes ornant la corbeille, à la forme de l'abaque, etc., mais aussi et peut-être surtout aux dimensions qui augmentaient les possibilités, plus réduites pour une colonnette d'autel ou un pilier-colonnette de chancel haut, plus importantes pour les exemplaires les plus gros qui sont ceux des différents ciboria. Si l'on continua à articuler sur les chapiteaux comme sur les bases des colonnes et colonnettes des éléments du vocabulaire architectural antique, des évolutions stylistiques notables sont évidemment sensibles dans le travail en relief de

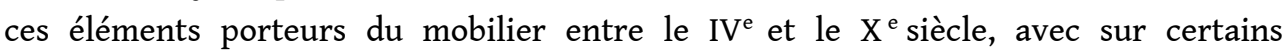
exemplaires l'ajout de motifs symboliques comme la croix, qui apparaissaient parfois aussi sur le fût des colonnes de ciborium, sinon lisses, torsadées ou cannelées et rudentées comme dans l'Antiquité.

8 Toutefois, ainsi que nous l'avons souligné plus haut, c'est sur les meubles ou les parties de meubles offrant des surfaces de matière plus étendues, planes pour les chancels et les ciboria ou légèrement incurvées pour les ambons, que purent se déployer largement moulures, gravures et sculptures, fréquemment rehaussées de couleur. La grande majorité des décors sculptés des plaques, épistyles et arcs étaient mis en page selon une grammaire ornementale directement héritée des pratiques antiques, impliquant le plus souvent des bordures, des encadrements saillants et moulurés, déterminant à leur tour des cartouches et des champs de forme quadrangulaire simple (rectangles plus ou moins allongés ou étirés en hauteur sur les plaques, où plusieurs se combinaient comme par exemple à Bayeux ${ }^{9}$, et piliers des barrières; trapèzes ou triangles des parapets d'accès aux ambons, comme celui de Saint-Jean de Maurienne ${ }^{10}$, etc.); ou à peine plus élaborée (demi-cercles, comme à Soyon ${ }^{11}$ et Saint-Raphaël ${ }^{12}$, ou rectangles échancrés à leur base pour les archivoltes des ciboria, ainsi celui de la cathédrale de $M e t \mathrm{z}^{13}$ ). Les moulures reprenaient les modénatures classiques (tores, scoties, rubans, doucines, cavets, gorges, bandeaux obliques, dentelures, perles et pirouettes, oves et dards, files de flots...). Au sein de ces cadres, s'épanouissaient des systèmes ornementaux relativement codifiés et par conséquent attendus comme tels sur un meuble donné. L'héritage clairement revendiqué se traduit sur les chancels par une évocation prégnante de ce qui en avait constitué le modèle: des barrières antiques, ajourées en grilles orthogonales ou obliques, maintenues par un cadre et des supports intermédiaires coiffés d'un pommeau. En ont résulté pendant toute la période étudiée dans le corpus SATHMA de multiples réseaux géométriques couvrants l'entièreté du champ des plaques (rarement sur les deux faces), dont certains - travaillés à jours ${ }^{14}$ répondaient pleinement au modèle idéal de l'époque romaine classique. Plus ou moins denses ou aérés, ces réseaux croisant, torsadant, nouant ou imbriquant comme une vannerie des formes simples (cercles adjacents ou sécants, carrés et losanges, polygones, fuseaux, écailles...) étaient pratiquement les mêmes que ceux qui gouvernaient les tracés des mosaïques de pavement de l'Antiquité tardive. Comme dans 
ces derniers, chaque forme dessinée par le réseau structurant pouvait être - ainsi par exemple sur la grande plaque de chancel de la cathédrale de Vence ou celle de SaintMartin d'Angers ${ }^{15}$-, emplie ou timbrée d'un motif issu du répertoire iconographique antique : élément végétal (feuille, fruit, rinceau, palmette, fleur ou fleuron, etc.), comme sur une plaque carolingienne de la cathédrale de $\mathrm{Die}^{16}$, géométrique ou figuré (animal, panier de pains ou de fruits, étoile, rosace tracée au compas, hélice, spirale, nœud, paire de colonnes, arc ou arcade...).

Mis à part quelques motifs nouveaux et caractéristiques comme les extrémités de tresses en têtes d'animaux que l'on observe sur le chancel de Saint-Pierre-auxNonnains, par exemple, l'apport «barbare» à ce vocabulaire et cette syntaxe " antiques» semble à rechercher en premier lieu dans les possibilités qu'offrait le traitement peu marqué de la gravure ou du relief méplat, adapté tant à l'épaisseur faible des plaques qu'à une évolution du goût entamée déjà au seuil de l'Antiquité tardive. Comme dans d'autres médiums artistiques tels que l'orfèvrerie ou l'enluminure, cette appétence nouvelle pour une représentation moins tridimensionnelle dans sa conception et dans sa lecture aboutit à un aplanissement et à une stylisation progressive de formes contenant des motifs épurés, à une perception nouvelle de la sculpture. Le rendu en fut certes souvent simplifié mais puissamment évocateur grâce à un contraste renforcé, presque dramatique, des ombres et des lumières. Et ce avant même que les réseaux directeurs des champs moulurés ne deviennent ces entrelacs touffus et ces tresses raffinées qui se diffusèrent largement depuis Rome à la faveur de la renouatio carolingienne. Les rubans qui dessinaient alors sur de faibles profondeurs des résilles d'entrelacs en relief méplat, aux subtils jeux d'ombre et de lumière, réunissaient majoritairement trois brins, certains étaient plats et rainurés, perlés, gemmés ou emplis de petites feuilles comme des galons (ainsi sur une plaque de chancel de Saint-Guilhem-le-Désert ${ }^{17}$ ), d'autres enfin semblaient des tores cordés ou torsadés (ainsi pour souligner les seuls rubans en diagonale de la plaque de chancel de Bayon-sur-Gironde) - et l'on retrouvait là des qualités évoquant singulièrement l'orfèvrerie "barbare ", ses sertissages cloisonnés, ornés de filigranes, de grènetis..., davantage encore quand les décors sculptés étaient rehaussés comme à l'origine de peinture colorée.

\section{L'affichage de motifs sculptés signifiants...}

10 Arcs et plaques constituant les autels, les ciboria, les chancels et les ambons affichaient une bonne part des messages visuels complexes portés par la sculpture tardo-antique et alto-médiévale, au premier titre desquels on classera l'expression, la symbolisation du divin. Le mobilier liturgique disposé autour de la table eucharistique et l'autel même étaient en effet assez logiquement les supports privilégiés de symboles ou de figures qui exaltaient la personne du Christ, promesse de salut éternel et verbe de Dieu. Le symbole le plus fréquent était la croix, latine ou grecque, aux branches pattées, qu'elle soit sculptée en relief méplat ou gravée, isolée (ainsi sur l'autel de Celeyran ${ }^{18}$ ) ou intégrée dans diverses compositions (ainsi la petite croix grecque à extrémités en spirales bouletées d'un fragment de chancel de Saint-Étienne de Lyon par exemple). Les grandes croix des plaques de chancel ou d'ambon évoquaient assez habituellement les croix de procession, gemmées et flanquées de l'alpha et de l'oméga suspendus à la traverse horizontale. Il en était de même pour les abréviations et monogrammes, tels le chrisme 
formé par les lettres chi-rhô (premières lettres du mot Xpıбtóc) ou les abréviations iotachi et tau-rhô (cette dernière dite aussi croix monogrammatique, avec souvent un rhô ouvert en R latin, ainsi qu'en offrait la façade des autels de Notre-Dame-du-Bourg à Digne et de Bagnols-sur-Cèze) (fig. 2). Alpha majuscule et oméga minuscule prenaient place entre les bras du chi (ainsi sur le grand chrisme gravé sur le fond de la mensa en sigma paléochrétienne de Saint-Pierre de Vienne ${ }^{19}$ ) ou sous la barre du tau (ainsi sur l'autel-cippe de Cassis dont la face antérieure arbore un chrisme sous deux croix monogrammatiques). À chacune de leurs très nombreuses occurrences, croix et chrismes ciselés proclamaient visuellement le nom du Christ dans l'espace ecclésial. La vigne constituait un autre rappel vivifiant du discours christique $(J n, 15,1)$, figuré dans ses enroulements couvrants chargés de grappes que picorent des colombes (ainsi sur une tranche latérale de la mensa de Saint-Victor de Marseille) et libérant des pampres ou en rinceaux de toutes tailles. Un peu plus rarement attesté, l'agnus dei évoquait symboliquement comme la croix le sacrifice, l'offrande pascale salvatrice dont témoignent l'exclamation de Jean Baptiste lors du baptême du Christ dans le Jourdain (Jn, 1, 29) et la victime immolée de l'Apocalypse de saint Jean (Ap, 5, 6-14) inspirée par l'agnelet de la Pâque juive. Pour finir, d'une manière extraordinaire et cette fois explicite, à l'opposé du parti aniconique précédent, existaient des bustes voire des figures en pied du Christ (ainsi sur une plaque du célèbre chancel de Saint-Pierre-auxNonnains à $\mathrm{Metz}^{20}$.

Fig. 2.

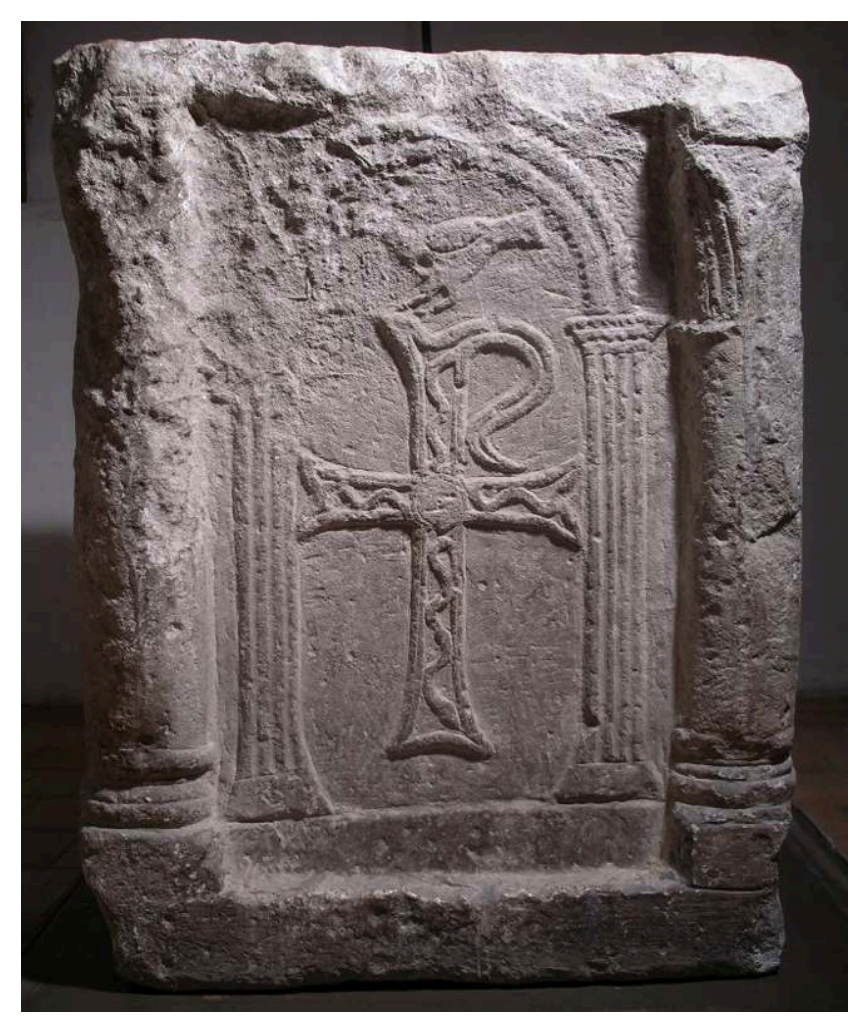

Autel-cippe en calcaire de Saint-Victor de Castel à Bagnols-sur-Cèze, conservé au Musée d'Art sacré du Gard, Pont-Saint-Esprit.

(C) X. Garrigou

11 Également présents dans l'iconographie funéraire de l'époque, ces motifs sculptés ou gravés dans la pierre ou le stuc des meubles liturgiques (chancel au seuil du sanctuaire, 
autel, ambon...) et qui symbolisaient ou représentaient le Christ sauveur, étaient le plus souvent tournés vers les fidèles, leurs destinataires privilégiés, mais ils étaient également présents sur la sculpture architecturale de l'église et sur de nombreux autres supports (sols de mosaïque, peintures et mosaïques murales, textiles, vaisselle liturgique, luminaire...). Leur répétition dans l'espace ecclésial constituait à l'évidence une des garanties de leur efficacité.

\section{... combinés à d'autres messages visuels}

Fig. 3.
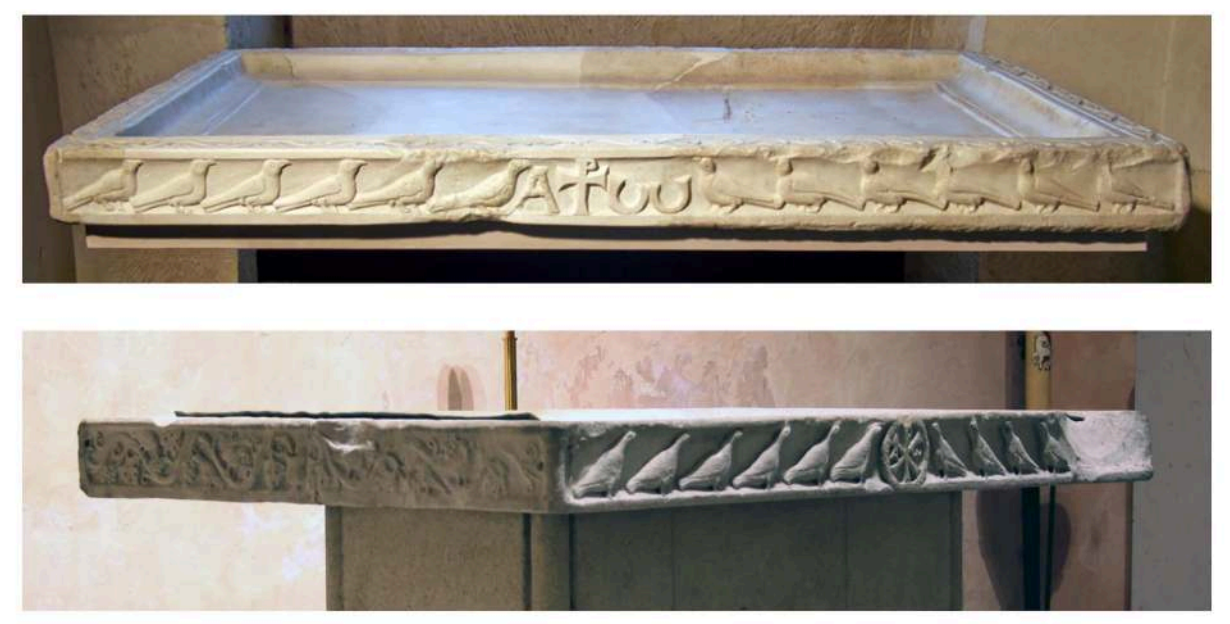

Mensa d'autel en marbre de Saint-Marcel de Crussol, conservée au Musée d'Archéologie nationale, Saint-Germain-en-Laye, et Mensa d'autel en marbre de Saint-Victor de Marseille.

(c) MAN @ R. Valette 
Fig. 4.

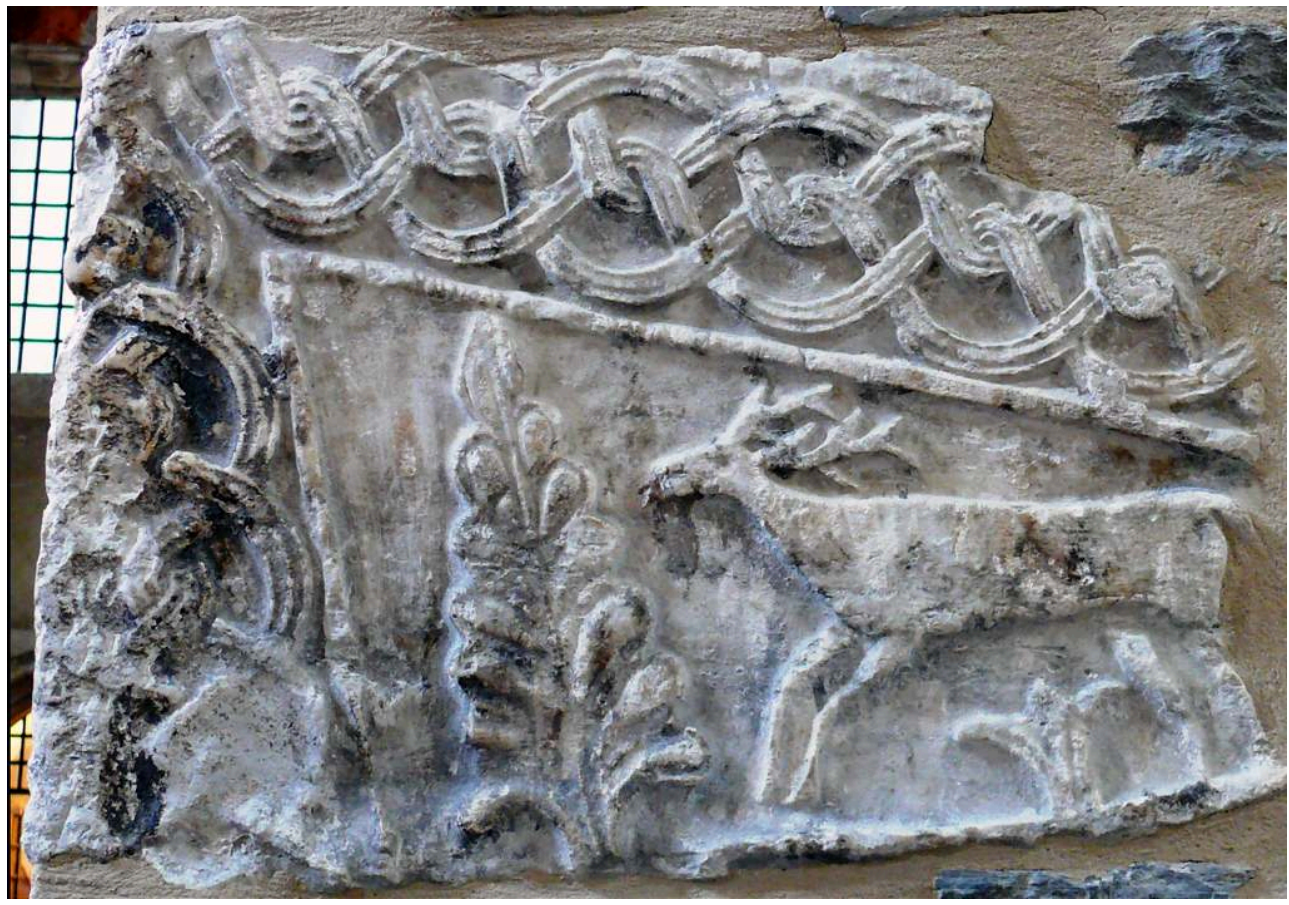

Plaque de parapet d'escalier de l'ambon de Saint-Jean de Maurienne, remployée dans un pilier.

(c) G. Mossot

12 Les symboles aniconiques et les quelques figures explicites du Christ étaient en général associées à d'autres motifs contribuant à les mettre en valeur, comme des arcs ou arcades, des piédestaux...; d'autres les dotaient de sens complémentaires. On trouvait ainsi par exemple les apôtres ou les fidèles symbolisés par des brebis, des colombes... en processions convergeant vers un motif christique central, croix, chrisme ou agneau (ainsi sur les plateaux d'autel de Saint-Victor de Marseille ${ }^{21}$ et de Saint-Marcel de Crussol $^{22}$ ou celui de Buoux ${ }^{23}$ ) (fig. 3) - comme le faisaient lors des cérémonies réelles, les clercs processionnant dans l'église et entrant à travers le chancel dans le chœur jusqu'à l'autel; à la façon aussi des fidèles se dirigeant vers l'ambon d'où résonnait la parole (c'est ce qu'illustrait le parapet d'escalier d'ambon de Saint-Jean de Maurienne ${ }^{24}$ ) (fig. 4). L'adoration, la vénération et, partant, la certitude du salut éternel étaient aussi des thèmes très fréquemment représentés selon des modalités déjà attestées dans le monde romain de l'Antiquité classique et tardive, influencé par la Perse sassanide : un motif trine, affrontant ou adossant deux animaux à un élément central (arbre de vie, vase ou coupe). On trouvait ainsi sur les chancels, les autels et les ambons des paires de colombes (ainsi sur deux des blocs de l'autel de Saint-Polycarpe ${ }^{25}$ ) ou de brebis, de cerfs (allusion au psaume 42), de paons, symboles d'éternité (ainsi sur l'ambon carolingien de l'̂̂le Barbe à Lyon ${ }^{26}$ ) (fig. 5), ou de lions, images de puissance, disposés de part et d'autre d'une croix, d'un chrisme, d'un arbre fructifère ou d'un buisson d'acanthe, d'une source d'eau, d'une fontaine, d'un vase d'où surgissait souvent la vigne-parole de Dieu, ou encore de la coupe du sacrifice eucharistique... De même que les signes éminents du Christ, des motifs animaliers symboliques isolés habitaient des rinceaux de vigne couvrant les grands champs ornementaux que l'on observe aux V $\mathrm{V}^{\mathrm{e}}$-VII ${ }^{\mathrm{e}}$ siècles (ainsi sur une plaque de chancel d'Alba ${ }^{27}$ ), comme ils le faisaient jadis sur les tapis de mosaïque antique à rinceaux habités, dont plaques et piliers de chancels constituent en quelque 
sorte une projection verticale. De la même manière, des animaux isolés, des arbres de vie poussant du sol ou dans un vase, des coupes où s'abreuvaient des oiseaux ou des quadrupèdes assoiffés, parsemaient parfois les réseaux et entrelacs géométriques évoqués plus haut pour les $\mathrm{VIII}^{\mathrm{e}}-\mathrm{X}^{\mathrm{e}}$ siècles. Les combinaisons extrêmement variées de motifs reflètent à la fois le savoir-faire des ateliers de sculpteurs, leur large répertoire et les choix réfléchis des commanditaires.

Fig. 5.

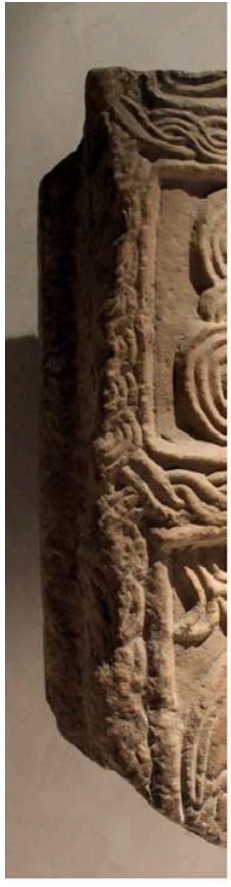

TRANChE

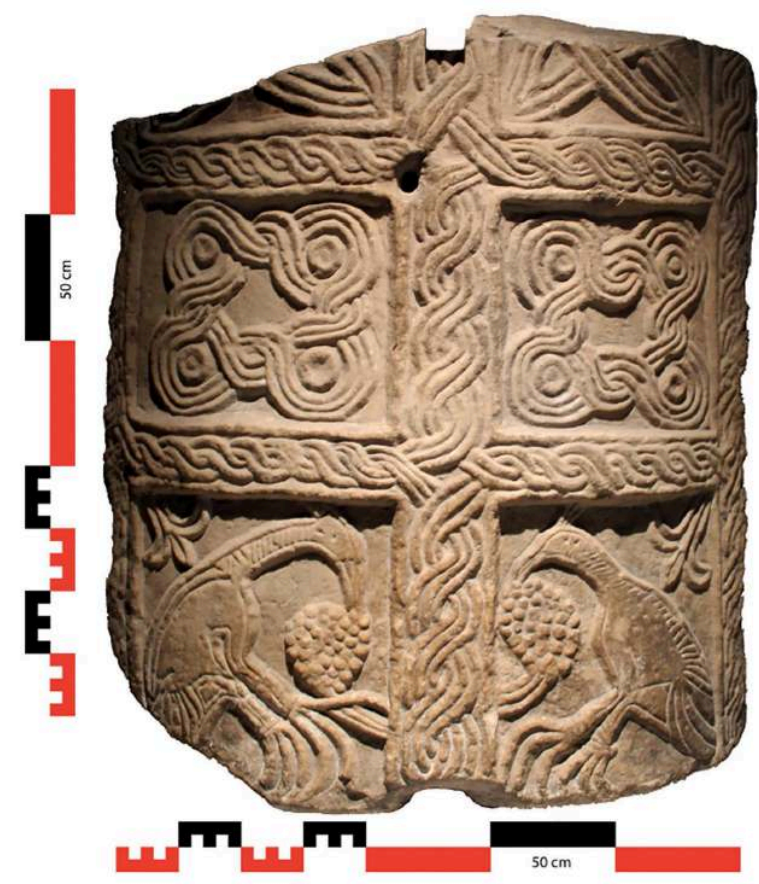

FACE A

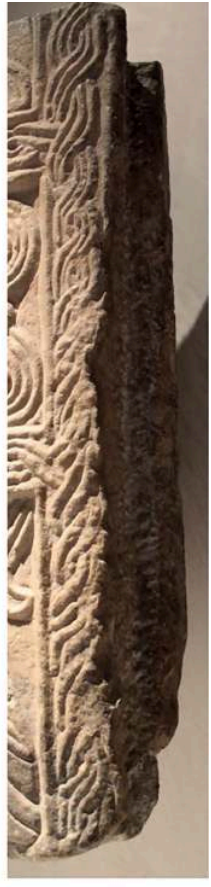

Tranche DROITE

Plaque de parapet de la plateforme de l'ambon de l'île Barbe à Lyon.

(c) J. Crochat

13 Les meubles sculptés dont certains fragments nous sont parvenus renseignent ainsi l'activité des tailleurs de pierre et des sculpteurs ainsi que la circulation de leurs produits. Quand on connait ou comprend leur emplacement d'origine, ils permettent encore de restituer l'organisation hiérarchisée de l'espace liturgique et son utilisation rythmée par leur décor sculpté, et de déceler certaines évolutions du rituel qui induisent une modification des aménagements matériels, alors que les sources écrites restent muettes à ce sujet. Enfin, si l'on devine ainsi de manière générale où se trouvaient les clercs et les fidèles au cours des cérémonies eucharistiques, ou encore les moines ou moniales au cours des offices, assez peu nombreux sont les documents qui permettent de mesurer le rôle des commanditaires. Même si ces cas sont relativement rares, chaque élément du mobilier pouvait néanmoins être le support d'une inscription de dédicace - celle du meuble ou celle de l'édifice entier - ou d'ex-voto. Le formulaire renseignait habituellement sur l'identité $\mathrm{du}$ ou des dédicants ou donateurs ecclésiastiques ou laïques ${ }^{28}$, la période de réalisation et quelquefois même nommait le meuble offert. Ces épigraphes n'étaient pas toujours lisibles, en particulier lorsqu'elles étaient gravées sur les autels qu'une nappe recouvrait pendant les célébrations et qui, 
le reste du temps, étaient peu accessibles à la lecture pour les fidèles contraints de demeurer à distance, en deçà du chancel. Ces inscriptions étaient de ce fait davantage destinées au clergé qui entourait l'autel qu'aux laïcs, même quand elles étaient exposées vers les fidèles. En outre, la compréhension de ces écrits dépendait du degré d'instruction des lecteurs potentiels. Pour les illettrés, qui en percevaient toutefois la sacralité et à qui on pouvait éventuellement les lire, une ou plusieurs lignes de lettres, gravées sur la tranche de la mensa d'un autel (ainsi sur ceux de Minerve ${ }^{29}$ au Ve siècle ou du $\mathrm{Ham}^{30}$ au VII ${ }^{\mathrm{e}}$ siècle) ou ciselées sur un chancel, ne signifiaient sans doute rien d'autre qu'une frise horizontale de petits motifs géométriques, une simple ornementation.

Autels, ciboria, cathèdres, chancels de chœur et parapets divers, ambons, tables secondaires, fonts baptismaux coiffés de dais...: le mobilier liturgique représente logiquement une large part des sculptures des $\mathrm{IV}^{\mathrm{e}}-\mathrm{X}^{\mathrm{e}}$ siècles publiées dans les quatre volumes du Recueil général des monuments sculptés en France pendant le haut moyen âge ${ }^{31}$, qui entrent aujourd'hui dans le corpus SATHMA. Chaque dispositif articulait une matérialité, une forme, un aspect visuel agençant dialectiquement ornements et images, et une fonction voire des fonctions rituelles. L'analyse des fragments multiples subsistant des différents meubles apporte des données sur leur élaboration technique, sur les sculpteurs qui en ont eu la charge, sur quelques commanditaires même, et plus généralement sur les utilisateurs de l'espace ecclésial, clercs et fidèles. Les rituels disposaient dans ces meubles d'écrins et de jalons spatiaux sculptés, parfois inscrits et souvent rehaussés de peintures s'accordant au reste de l'ambiance colorée de l'édifice cultuel. Riches d'enseignements variés pour le chercheur, les messages visuels portés par ces meubles loquaces étaient destinés aux différents acteurs en présence. Les formes des vestiges conservés et les traces de leur ancrage au sol font percevoir des lignes directrices ou séparatrices dans la nef, le sanctuaire et certaines annexes liturgiques comme le baptistère. En regroupant une myriade d'éléments sculptés dispersés dans toute la France dans un système informatique offrant tous les avantages des humanités numériques, le corpus en ligne SATHMA sera le terreau de comparaisons encore inédites, de regroupements de pièces, voire dans certains cas de reconstructions graphiques d'ensembles bien identifiés, comme celles du chancel pergula de Pouthumé (fig. 1) ou tout dernièrement du ciborium baptismal de Mariana ${ }^{32}$. Associés à ces images recouvrées, les dispositifs identifiés in situ permettront in fine de reconnaître usages et fonctions et, partant, de mieux restituer virtuellement les mouvements principaux d'une liturgie qui évolua entre le $\mathrm{IV}^{\mathrm{e}}$ et le $\mathrm{X}^{\mathrm{e}}$ siècle et dont les textes n'expliquent guère les détails ou les variations. 


\section{NOTES}

1. Catherine Metzger, «Le mobilier liturgique », sous la direction de Noël Duval, Naissance des arts chrétiens, Paris, Imprimerie nationale, 1991, pp.256-267 ; Ead., "Installations liturgiques en Gaule ", Hortus Artium Medievalium, t. 5, 1999, pp. 41-44.

2. Voir par exemple Anne-Bénédicte Mérel-Brandenburg, «Le sanctuaire en Gaule (IV e_ VIII ${ }^{\mathrm{e}}$ siècles)", Hortus Artium Medievalium, t. 15, 2009, pp. 77-86; Thomas Creissen, «L'aménagement du sanctuaire dans les églises de France avant l'an Mil », ibidem, pp. 87-103.

3. Ce phénomène de préfabrication s'observe pour l'ensemble de la sculpture architecturale des églises qui était parfois commandée à grande distance ou importée. C'est ce qui faisait vivre en partie certaines carrières impériales de marbre du bassin méditerranéen. En témoigne en particulier la cargaison composée de colonnes destinées à la nef d'un bâtiment et son mobilier liturgique (chancel haut, autel, ciborium et ambon à double escalier presque complet), découverte en 1967 au sud-est de la Sicile dans l'épave de Marzamemi, dont l'étude a repris en 2013. Voir Gerhard Kapitän, «Elementi architettonici per una basilica dal relitto navale del VI secolo di Marzamemi (Siracusa) », La Sicilia dal tardoantico ai Normanni, Corsi di cultura sull'arte ravennate e bizantina, XXVII, Ravenne, Edizioni del Girasole, 1980, pp. 71-136 ; Justin Leidwanger, Sebastiano Tusa, « Relitto di Marmazemi. Metti una chiesa da... Ikea », Archeologia Viva, t. 36/184 ns, juilletaoût 2017, pp.32-40. Pour resituer enfin les trouvailles de Marzamemi dans le contexte du commerce international du marbre en Méditerranée, grâce à cinq autres épaves d'Italie et de Turquie qui transportaient des colonnes pour des églises « en kit », voir Elena Flavia Castagnino Berlinghieri, Andrea Paribeni, «Byzantine Merchant Ships and Marble Trade: New data from Central Mediterranean ", In Poseidon's Realm XV: Byzantium at Sea. Innovation and Tradition, Wien 2010 (Skyllis: Zeitschrift für Unterwasserarchäologie, 11-1), Vienne, 2011, pp. 64-75.

4. Voir par exemple Élisabeth Châtel, "Autels-Cippes de Septimanie ", Gaule mérovingienne et monde méditerranéen, Actes des IX Journées d'archéologie mérovingienne, Lattes, 24-27 septembre 1987, sous la direction de Christian Landes, Lattes, Musée archéologique, 1988, pp. 115-123.

5. Ainsi par exemple celui de Saint-Marcel-de-Careiret, remployé, sculpté et inscrit à la fin du $\mathrm{VI}^{\mathrm{e}}$ siècle ou au début du siècle suivant, voir A.-B. Mérel-Brandenburg, La sculpture de l'Antiquité tardive en Languedoc méditerranéen et Roussillon (IV ${ }^{e}$-VIII ${ }^{e}$ s.), Thèse de doctorat, Université Paris IVSorbonne, sous la direction de François Baratte, 2007, pp.338-340, pl. 152 ; Yumi Narasawa, Les autels chrétiens du Sud de la Gaule ( $\mathrm{V}^{e}$-XII ${ }^{e}$ siècles), Turnhout, Brepols (Bibliothèque de l'Antiquité tardive, 27), 2015, pp. 303-306.

6. Anne Flammin, «La sculpture du Haut Moyen Âge en Poitou-Charentes: les récentes découvertes d'Usseau (Deux-Sèvres) et de Pouthumé (Vienne)», Wisigoths et Francs autour de la bataille de Vouillé (507). Recherches récentes sur le haut Moyen Âge dans le Centre-Ouest de la France, Actes des XVIII Journées internationales d'archéologie mérovingienne, Vouillé et Poitiers (Vienne, France), 28-30 septembre 2007, sous la direction de Luc Bourgeois, Saint-Germain-en-Laye, AFAM (Mémoires publiés par l'AFAM, 22), 2010, pp. 237-249.

7. Manuel Moliner, «La memoria de la basilique paléochrétienne de la rue Malaval à Marseille, premières approches ", Saint-Victor de Marseille, études archéologiques et historiques. Actes du colloque Saint-Victor, Marseille, 18-20 novembre 2004, sous la direction de Michel Fixot, Jean-Pierre Pelletier, Turnhout, Brepols (Bibliothèque de l'Antiquité tardive, 13), 2009, pp. 107-123.

8. Yann Codou, "Le monument funéraire carolingien de Saint-Pons de Cimiez", Histoire et archéologie de la Provence antique et médiévale. Hommages à Jean Guyon, sous la direction de Yann Codou, Marc Heijmans, Marseille, Fédération historique de Provence (Provence historique, t. 61), 2011, pp. 279-295, avec la bibliographie antérieure. 
9. On trouve fréquemment sur les plaques des $\mathrm{VIII}^{\mathrm{e}}-\mathrm{X}^{\mathrm{e}}$ siècles une large bande sommitale en légère saillie, peut-être héritée des modèles paléochrétiens sommés de mains courantes, et quelquefois des bandes latérales le long des piliers, encadrant le champ principal en rectangle barlong sculpté encore en retrait. C'est le cas de la plaque de Bayeux : Florence Delacampagne, Xavier Savary, «Bayeux. Une plaque de chancel carolingien », Bulletin monumental, t. 167-4, 2009, pp. 357-358, fig. 1 .

10. Cette plaque fragmentaire de parapet d'ambon d'époque carolingienne, remployée dans le cinquième pilier nord de la cathédrale, était trapézoïdale.

11. Micheline Buis, «Les sculptures carolingiennes de l'église de Soyons (Ardèche) », Revue du Vivarais, t. 2, 1984, pp. 65-72.

12. Voir Th. Creissen, op. cit. note 2, p. 97 et fig. 23.

13. May Vieillard-Troiekouroff, «Les chancels et le ciborium de la cathédrale de Metz de l'évêque Chrodegang ", Monuments et Mémoires Fondation Eugène Piot, t. 70, 1989, pp. 55-68.

14. Ainsi par exemple les deux plaques fragmentaires à entrelacs ajourés remployées à la crypte de la cathédrale de Clermont-Ferrand, voir en dernier lieu Pascale Chevalier, "Les vestiges sculptés de la cathédrale ", Clermont, l'âme de l'Auvergne, sous la direction de Mgr. Hippolyte Simon, Strasbourg, La Nuée bleue (coll. « La grâce d'une cathédrale »), 2014, pp. 142-147.

15. M. Vieillard-Troïekouroff, "La sculpture en Neustrie », Beihefte der Francia, t. 16, $\mathrm{n}^{\circ}$ 2, 1989, pp. 225-258, en particulier p. 253 et fig. 21 p. 252. L'entrelacs de cercles jointifs couvrant le champ y dessine des carrés sur la pointe curvilignes. Les cercles enferment des hélices à bouton central, les carrés sur la pointe et les écoinçons en bordure contiennent des fleurons trifides.

16. La trame du champ entrelace de petits cercles qui dégagent des octogones curvilignes. Cercles et octogones enferment alternativement des fleurs à six pétales posées sur un disque, des grappes de raisin, un bouton de fleur, une volute.

17. Géraldine Mallet, « Nouveau regard sur les fragments de chancels de l'ancienne abbaye SaintSauveur de Gellone à Saint-Guilhem-le-Désert (Hérault) », Le chancel de Saint-Pierre-aux-Nonnains. Actes du colloque, Metz, Musée de La Cour d'Or, 28-29 avril 2017, sous la direction d'Anne Adrian, Cinisello Balsamo, Silvana editoriale, 2021, pp. 120-131.

18. A.-B. Mérel-Brandenburg, op. cit. note 5, pp.337-338, pl.150; Y. Narasawa, op. cit. note 5, pp. 338-340.

19. Monique Jannet-Vallat, Roger Lauxerois, Jean-François Reynaud, Vienne (Isère) aux premiers temps chrétiens, Paris, Imprimerie nationale (Guides archéologiques de la France), 1986, p. 36. Le chrisme " orfévré » est gravé et champlevé pour les gemmes, alternativement rectangulaires et ovales, dans une couronne tressée, l'alpha et l'oméga se présentent comme des pendeloques suspendues à une chaînette.

20. Voir en dernier lieu Le chancel de Saint-Pierre-aux-Nonnains, op. cit. note 15.

21. Y. Narasawa, op. cit. note 5, pp. 190-194. Sur une des longues tranches, des colombes convergent vers un chrisme, sur la tranche opposée des brebis se dirigent vers un agneau divin. Voir aussi Ead., "L'autel paléochrétien de Saint-Victor et les autels apparentés: essai de chronologie ", Saint-Victor de Marseille, études archéologiques et monumentales. Actes du colloque SaintVictor - Marseille, 18-20 novembre 2004, sous la direction de Michel Fixot, Turnhout, Brepols (Bibliothèque de l'Antiquité tardive, 12), 2009, pp. 45-68.

22. Y. Narasawa, op. cit. note 5 , pp. 380-381. Sur une des longues tranches du plateau, deux théories de six colombes et sur la tranche opposée deux théories de six brebis sortant de deux villes (Jérusalem et Bethléem) cheminent vers une croix monogrammatique en semi-méplat, cantonnée de l'alpha et de l'oméga. L'autel en marbre est conservé au Musée des Antiquités nationales, Inventaire $n^{\text {os }}$ MAN 19589, 20300, 20580 et 90966.

23. Deux paires d'agneaux se dirigent ou adorent à genoux une croix et, sur la face contiguë de la tranche de cette mensa des VIII ${ }^{\mathrm{e}}-\mathrm{X}^{\mathrm{e}}$ siècles, deux paires de paons ou de colombes vénèrent une croix monogrammatique. Voir Guy Barruol et Y. Codou, «Le mobilier liturgique », cat. d'exp., 
D'un monde à l'autre. Naissance d'une chrétienté en Provence, $I V^{e}-V^{e}$ siècle, sous la direction de Jean Guyon, Marc Heijmans, Musée de l'Arles antique, 15 septembre 2001-6 janvier 2002, Arles, Éditions du Musée de l'Arles antique, 2001, pp. 167-168; et Y. Codou, ibidem, p. 225.

24. Sa large bordure de cercles entrelacés encadre en effet un cerf se dirigeant vers un arbuste dont il compte se nourrir, comme le fidèle le faisait de la parole de Dieu.

25. Un des deux autels romans mettant en œuvre des remplois d'époque carolingienne, est soutenu à l'arrière par deux plaques identiques ou les deux extrémités d'une seule longue plaque (probablement de chancel, s'il ne s'agissait pas d'une face de cuve de sarcophage) : un troisième arc pourrait être dissimulé par le bloc à entrelacs placé au-devant dans le montage actuel. Sur chacune, un arc sur colonnes torses abrite une demi-fleur à 8-9 pétales et une tresse nouée en croix au pied de laquelle deux colombes picorent des grains de raisin.

26. Les deux paons picorant une grappe sont affrontés à un ruban tressé d'où émergent les grappes et qui dans le quadrillage de la plaque rappelle la croix-arbre de vie. Voir Jessy Crochat, Le mobilier liturgique dans les diocèses médiévaux de Grenoble, Lyon et Vienne, IVe-XIe siècle, Mémoire de Master 2, sous la direction d'Anne Baud, Université Lumière-Lyon 2, 2015, vol. 1. Inventaire du mobilier et des vestiges archéologiques, notice 10, pp. 48-50, fig. p. 48.

27. Yves Esquieu, «Les plaques ornées d'entrelacs provenant d'Alba », Revue du Vivarais, t. 79, 1975, pp. 129-134, fig. 4.

28. Ce nom peut être remplacé par une allusion, comme le donateur anonyme indignus peccato[r...] de la plaque de chancel carolingienne de Carpentras (l'inscription courait sur au moins trois côtés de la bordure), voir Micheline Buis, "Note sur une dalle carolingienne de Carpentras ", Archéologie du Midi médiéval, t. 13, 1995, pp. 239-242.

29. Joseph Braun, Der christliche Altar in seiner geschichtlichen Entwicklung, t. 1, Arten, Bestandteile, Altargrab, Weihe, Symbolik, Munich, Alte Meister Guenther Koch \& Co., 1924, p. 300 ; A.-B. MérelBrandenburg, op. cit. note 5, pp. 321-322 ; Y. Narasawa, op. cit. note 5, pp. 209-211.

30. Amédée Léchaudé d'Anisy, "Quelques mots sur l'autel du Ham », Mémoires de la société des antiquaires de Normandie, t. 17, 1847, pp. 213-220.

31. Denise Fossard, M. Vieillard-Troïekouroff, É. Chatel, Recueil général des monuments sculptés en France pendant le haut Moyen Âge (IV ${ }^{e}-X^{e}$ siècles), 1. Paris et son département, Paris, CTHS, 1978 ; É. Chatel, idem, 2. Isère, Savoie, Haute-Savoie, 1981 ; Jacques Sirat, M. Vieillard-Trö̈ekouroff et É. Chatel, idem, 3. Le Val-d'Oise et les Yvelines, 1984 ; Christophe Deroo, Marcel Durliat, Maurice Scelles, idem, 4. Haute-Garonne, 1987 (Mémoires de la section d'archéologie et d'histoire de l'art, 1-4).

32. On doit l'étude du ciborium de Mariana à Anne Flammin et la restitution graphique à cette dernière et à Gérard Carpentier. Voir Mariana. L'évêché et les édifices de culte du V ${ }^{e}-X V I^{e}$ siècle, sous la direction de Daniel Istria, Rome, École Française de Rome (CEFR, 574), 2020.

\section{RÉSUMÉS}

Au sein de l'espace ecclésial, le mobilier liturgique - autel, ciborium, cathèdre, chancel, ambon était le lieu de sculptures au contenu plutôt aniconique. Les combinaisons de décors reprennent des répertoires à la mise en page caractéristique. Ciselure, gravure, champlevé, relief méplat ou travail ajouré se développaient sur les limites du chœur, renforçaient le sens de l'autel du sacrifice, le siège du célébrant, l'estrade d'où résonnait la parole... Les rituels disposaient 
d'écrins, de jalons sculptés, rarement inscrits, souvent rehaussés de peintures s'accordant à l'ambiance colorée de l'église. Riches d'enseignements pour le chercheur, ces meubles loquaces portaient des messages visuels symboliques. Les formes des vestiges et les traces de leur implantation dessinent des lignes fonctionnelles directrices ou séparatrices. On y reconnaît les mouvements d'une liturgie qui évolua entre le $\mathrm{IV}^{\mathrm{e}}$ et le $\mathrm{X}^{\mathrm{e}} \mathrm{s}$. et dont les textes expliquent peu les détails ou variations.

Within the ecclesial space, sculptures with mainly aniconic content were found on the liturgical furniture: altar, ciborium, cathedra, chancel, ambo, and so on. The combinations of decorations featured elements of repertoires with characteristic layouts. Chasing, engraving, champlevé, basrelief and openwork spread over the limits of the choir, reinforcing the significance of the sacrificial altar, the seat of the celebrant and the dais from which the Word resounded. The rituals had settings, sculpted milestones that were rarely inscribed and often enhanced by paintings that matched the colourful atmosphere of the church. Rich in information for the researcher, the loquacious pieces of furniture bore symbolic visual messages. The forms of the vestiges and the traces of their layout mapped out functional guidelines or dividing lines. In them, the movements of a liturgy that evolved between the fourth and tenth centuries may be recognised, one whose texts explain few of its details and variations.

\section{INDEX}

Mots-clés : sculpture, autel, chancel, ambon, croix, chrisme, entrelacs, espace ecclésial, haut Moyen Âge

Keywords : sculpture, altar, chancel, ambo, cross, chrism, interlacing, ecclesial space, Early Middle Ages

\section{AUTEUR \\ PASCALE CHEVALIER}

Pascale Chevalier est Maître de conférences HDR en Histoire de l'art et Archéologie médiévale, Université Clermont Auvergne et Directrice adjointe de l'UMR 6298 ARTEHIS. Son travail de Doctorat en Histoire et Civilisation de l'Antiquité, obtenu en 1991 sous la direction de Noël Duval à Paris IV-Sorbonne, s'intitulait : L'architecture paléochrétienne de la province romaine de Dalmatie [en dehors de Salona] - IV $V^{e}-\mathrm{VI}^{e}$ s. En 2021, elle obtient une Habilitation à diriger les recherches de l'Université Clermont Auvergne, avec son travail, Recherches archéologiques entre Antiquité et Moyen Âge, de la France aux confins de l'Orient méditerranéen, vol. inédit « Tempore episcoporum » - réflexions sur l'Istrie du VIe siècle.

Pascale Chevalier is a lecturer at the Université Clermont Auvergne accredited to supervise research in art history and medieval archaeology and assistant director of UMR 6298 ARTEHIS. She obtained her PhD from Paris IV-Sorbonne in 1991. Her thesis, supervised by Noël Duval, was titled "L'architecture paléochrétienne de la province romaine de Dalmatie [en dehors de Salona] IV ${ }^{e}-V I I{ }^{e}$ s." In 2021 she obtained an accreditation to supervise research at the Université Clermont Auvergne with her work on sixth-century Istria. 